\title{
Evaluation of the impact of residual stresses in crack initiation with the application of the Crack Compliance Method Part I, Numerical analysis
}

\author{
G. Urriolagoitia-Sosa ${ }^{a}$, B. Romero-Ángeles ${ }^{b}$, L. H. Hernández-Gómez ${ }^{c}$, \\ G. Urriolagoitia-Calderón ${ }^{d}, J$ J. A. Beltrán-Fernández ${ }^{\mathrm{e}}$, C. Torres-Torres ${ }^{f}$ \\ ${ }^{1}$ Instituto Politécnico Nacional, Sección de Estudios de Posgrado e Investigación, \\ Escuela Superior de Ingeniería Mecánica y Eléctrica, Unidad Profesional "Adolfo López Mateos" \\ Edificio 5, 2do Piso, Col. Lindavista, CP 07738, México, D. F. México \\ aguiurri@hotmail.com, 'romerobeatriz97@hotmail.com, 'luishector56@hotmail.com, \\ durrio332@hotmail.com, ejbeltranf@hotmail.com, ${ }^{\mathrm{f}}$ crstorres@yahoo.com.mx
}

Keywords: Crack initiation, residual stress, crack compliance method, modified SEN specimen.

\begin{abstract}
The understanding of how materials fail is still today a fundamental research problem for scientist and engineers. The main concern is the assessment of the necessary conditions to propagate a crack that will eventually lead to failure. Nevertheless, this kind of analysis tends to be more complicated, when a prior history in the material is taken into consideration and it will be extremely important to recognize all the factors involved in this process. In this work, a numerical simulation of the introduction of residual stresses, which change the crack initiation conditions, in a modified compact tensile specimen to change the condition of crack initiation is presented. Four numerical analyses were carried out; an initial evaluation was performed in a specimen without a crack and it was used for the estimation of a residual stress field produced by an overload; three more cases were simulated and a crack was introduced in each specimen $(1 \mathrm{~mm}, 5 \mathrm{~mm}$ and $10 \mathrm{~mm}$, respectively). The overload was then applied to set up a residual stress field into the component; furthermore, in each case the crack compliance method (CCM) was applied to measure the induced residual stress field. By performing this numerical simulation, the accuracy of the crack compliance method can be evaluated. On the other hand, elastic-plastic finite element analysis was utilized for the residual stress estimation. The numerical analysis was based on the mechanical properties of a biocompatible material (AISI 316L). The obtained results provided significant data about diverse factors, like; the manner in which a residual stress field could modify the crack initiation conditions, the convenient set up for induction of a beneficial residual stresses field, as well as useful information that can be applied for the experimental implementation of this research.
\end{abstract}

\section{Introduction}

It has been well documented that development of failure could be divided in two basic parts, initiation and spread [1]. Additionally, there is a great number of external and internal factors that contribute to the nucleation and propagation of a crack [2]. Slip bands or dislocations and surface scratches can be considered as internal effects, as external factors are considered the effect of forces and deformations. Nevertheless, when the development, performance and effect of a crack are analyzed, prior history in the material it is not considered extensively or in a sufficient manner. To consider prior history in the component raises the difficulty of the problem in a substantial way. This is why the simplest way to analyze failure and its consequences is to consider the specimen free of previous history. But on the other hand, the manufacture of components will always leave 
inside the material an induction of a stress or strain field and this field will interact with the development of all sorts of defects [3].

The induction of previous history into the component is based in the application of an external agent above the yielding strength of the material. The introduction of previous history can be divided in two groups, by homogenous loading or by non-homogenous loading. The consequence of a homogenous loading derives into strain hardening and Bauschinger effect, and such consequences are the introduction of residual stresses. In both cases, the consequences of the application and removal of the external agent could contribute into the material in a beneficial and/or detrimental manner. Strain hardening and Bauschinger effect can be found in the material at the same time, if the component has been strengthen by tensile strain hardening, Bauschinger effect (that is a reduction of the yield strength of the material) will be found in the compressive behavior and vice versa. In relation to residual stresses, they are also detrimental and beneficial, as tensile and compressive stresses are applied together and auto-equilibrate them self [4]. So, in the process of manufacturing pieces and components it is very important to identify the outcome that a particular process of fabrication could add to the material.

In this paper, it is presented the numerical simulation of the introduction of a residual stress field to modify the strength of the material. It can improve the mechanical resistance of the component by a tensile overload, which at the beginning of it action can propitiate the nucleation or propagation of a crack, but when the application of the external agent is ended it would leave a beneficial residual stress field.

\section{The Crack Compliance Method [4]}

In this section a brief summary of the theory relative to the CCM is presented. Let the unknown residual stress distribution in the beam be represented by the summation of an $n^{\text {th }}$ order polynomial series as:

$$
\sigma_{y}(x)=\sum_{i=0}^{n} A_{i} P_{i}(x)
$$

where $A_{i}$ are constant coefficients and $P_{i}$ are a power series, $x^{0}, x^{1}, x^{2}, \ldots x^{n}$ etc. The strain $\varepsilon(a, s)$ (where $a=$ crack length and $s=$ is the distance between the location of the strain gauge and the crack plane) due to the stress fields $P_{i}(x)$ is known as the compliance function $C_{i}\left(a_{j}, s\right)$ and is given by:

$$
C_{i}\left(a_{j}, s\right)=\frac{1}{E^{\prime}} \int_{0}^{a_{j}} K_{I}(a) Z(a) d a
$$

$K_{I}(a)$ is the stress intensity factor due to the residual stress field when the crack depth in the beam is equal to $a$ and $K_{I F}(a)$ is the stress intensity factor corresponding to the same depth due to a pair of virtual forces $F$ applied tangentially at a position on the beam where strain measurements will be taken during the $C C M$ cutting of the slot (where $Z(a)$ is a geometry dependant function (equation (3)):

$$
Z(a)=\frac{\partial^{2} K_{I F}(a, s)}{\partial F \partial s}
$$

By following the weight function approach, $K_{I}(a)$ and $K_{I F}(a)$ can be expressed as:

$$
\begin{aligned}
K_{I}(a) & =\int_{0}^{a} h(x, a) \sigma_{y}(x) d x \\
K_{I F}(a) & =\int_{0}^{a} h(x, a) \sigma_{y F}(x) d x
\end{aligned}
$$

where $\sigma_{y F}(x)$ is the stress field due to the virtual force $F$. Once the $C_{i}(a, s)$ solutions are determined the expected strain due to the stress components in equation (1) can be obtained as:

$$
\varepsilon\left(a_{j}, s\right)=\sum_{i=0}^{n} A_{i} C_{i}\left(a_{j}, s\right)
$$

The unknown terms $A_{i}$ are determined so that the strains given by equation (6) match those strains measured in the experiment during cutting i.e., $\varepsilon\left(a_{j}, s\right)_{\text {actual }}$. In order to minimise the average error over all data points for an $n^{\text {th }}$ order approximation, the method of least squares is used to obtain the values of $A_{i}$. The number of cutting increments $m$ is therefore chosen to be greater than the order of the polynomial, i.e. $m>n$. 
This work used $n=7$ with 8 constants $A_{i}$ and $m=9$, this being the number of experimental slot cutting depths at which strain readings were collected. The least square solution is obtained by minimising the square of the error relative to the unknown constant $A_{i}$, This gives a linear set of simultaneous solutions from which $A_{i}$ values are determined and equation (1) is then used to determine the residual stress distribution.

\section{Test specimen}

For this research it has been chosen a keyhole specimen, which is a modification of a compact tension specimen (ASTM standard E 647-91) with special requirements (ASTM E 399) (Fig. 1) [5]. The main characteristic of the component is a hole inducted at the notch tip. When a tensile load is applied which is large enough to produce plastic deformation at the stress concentration (point A) while the surface surrounding the material remains elastic, so when removing the effects of the load a compressive residual stress remains at the concentration point?

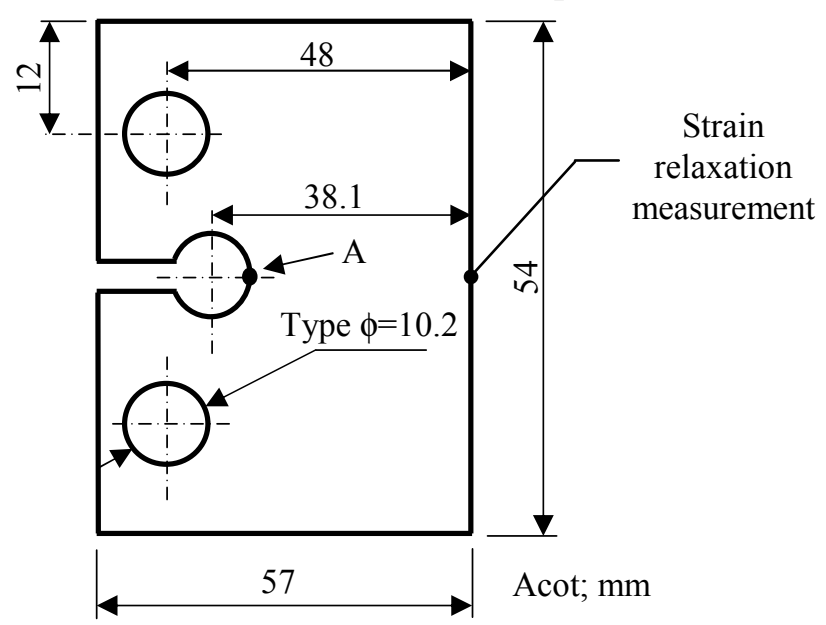

Figure 1.- Modified compact tensile specimen SEN

\section{Statement of the problem and numerical simulation}

The research presented in this paper is aimed to establish the effect of prior history in the development of a crack and how a beneficial residual stress field could increase the mechanical resistance of the material. Four cases of study are presented in this research. The mechanical properties employed for the numerical analysis were the ones obtained by four point bending tests performed on beams manufactured from a stainless steel AISI 316L (Fig. 2a) [6]. The elastic properties of the material were set up as follows; Young's modulus of $190000 \mathrm{~N} / \mathrm{mm}^{2}(E)$ and Poisson ratio of $0.28(v)$. For the elasto-plastic condition a kinematic hardening rule was applied and the mechanical properties were introduced in a tabular manner (Fig. 2b and Table 1).
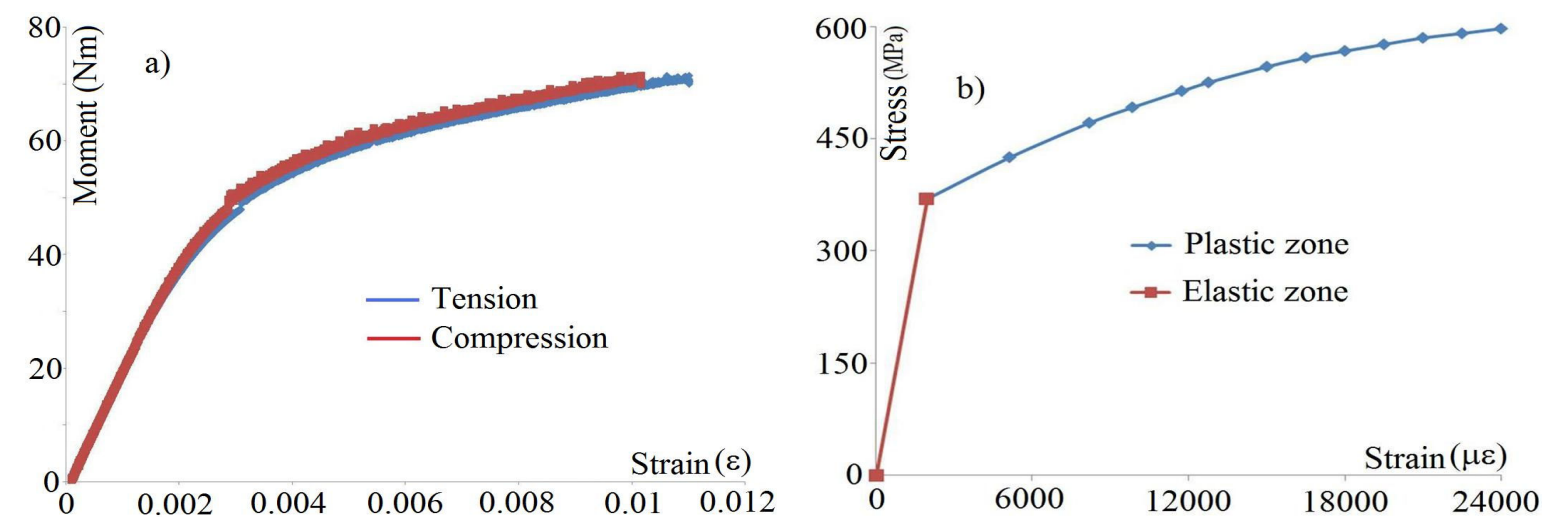

Figure 2.- Mechanical properties stainless steel AISI $316 \mathrm{~L}$

a) Bending test data used to obtain the stress-strain curve. b) AISI $316 \mathrm{~L}$ stress-strain curve. 
Table 1.- Stress-strain data used for the numerical simulation

\begin{tabular}{||c|c||}
\hline Stress (MPa) & Strain \\
\hline \hline 370 & 0.001947 \\
\hline 425 & 0.005104 \\
\hline 471 & 0.008171 \\
\hline 491.7 & 0.009828 \\
\hline 513.5 & 0.011726 \\
\hline 525 & 0.012756 \\
\hline 546 & 0.015000 \\
\hline 558 & 0.016500 \\
\hline 567 & 0.018000 \\
\hline 575.8 & 0.019500 \\
\hline 584.5 & 0.021000 \\
\hline 590.5 & 0.022500 \\
\hline 596.85 & 0.024000 \\
\hline
\end{tabular}

The numerical simulation was performed in a symmetrical manner and all the specimens were modeled in 2D, to save computational resources (Fig. 3). Quadratic order elements (Plane 183) were used with 8 nodes and plane stress analysis was performed. The specimen was loaded (in all four cases) in tension and the force was uniformly distributed in 17 nodes on the loading keyhole with a magnitude of $100 \mathrm{~N}$ each (Fig. 3a). The base of the specimen was specially prepared with a rectangular zone (elements dimensions $0.5 \mathrm{~mm}$ width by $1 \mathrm{~mm}$ large), this zone will be employed to simulate the introduction of a slot and to evaluate the $C C M$ and strain relaxation data will be obtained at the rear node of the base line (Fig. 3). Boundary conditions were applied at the bottom line in a symmetrical manner. The lack of application of boundary conditions at the near end of the bottom line is to simulate the introduction of a crack (Fig.3a) and this lack on the application of symmetry will depend on the length of the crack to be simulated. The introduction of the residual stress will be carried out by removing the effect of the load and once again resolving it (Fig. $3 b$ ).
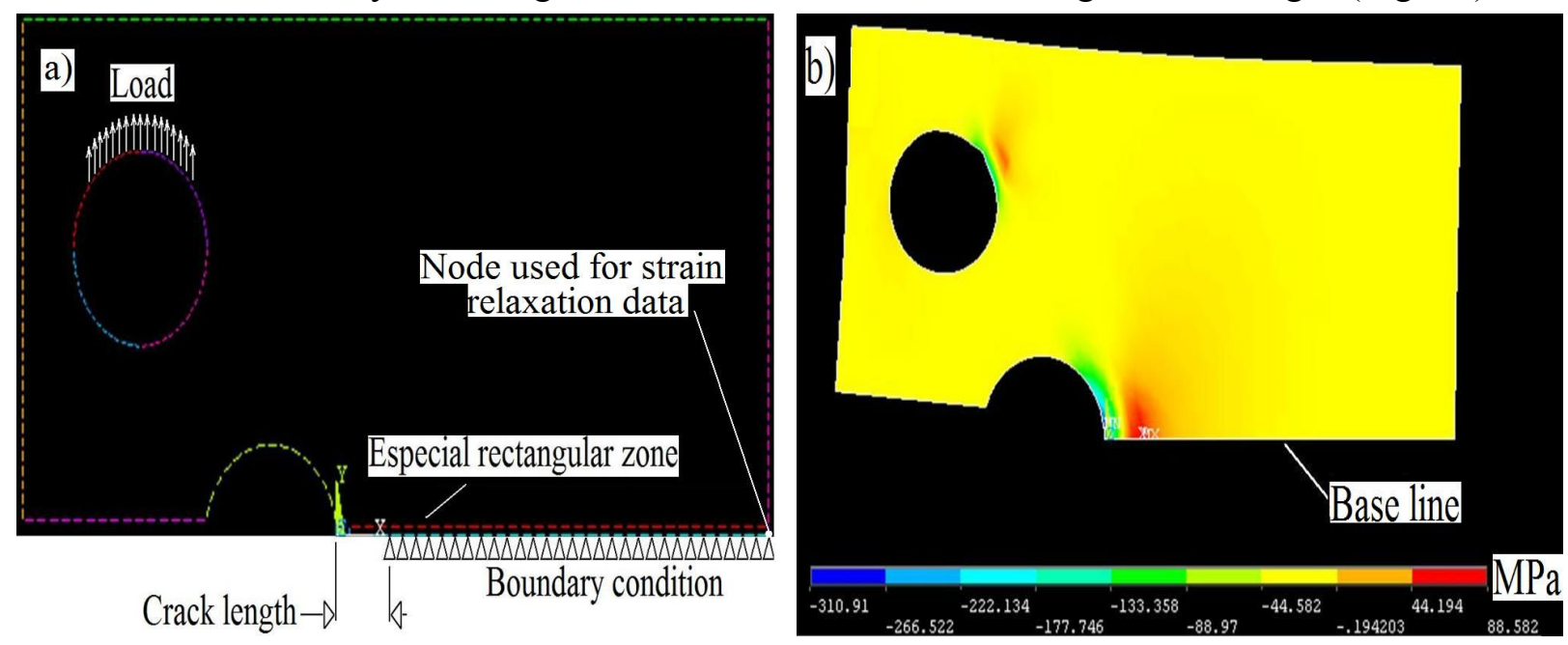

Figure 3.- Modified compact tensile specimen SEN modeled

a) Numerical model. b) Residual stress field for specimen without a crack.

\section{Numerical case of study and results}

The first case of study is the introduction of a residual stress field into a specimen free of a crack. For the next three cases of study, an analysis was performed on the effect of the introduction of a crack with different lengths $(1 \mathrm{~mm}, 5 \mathrm{~mm}$ and $10 \mathrm{~mm}$, respectively). The residual stress fields obtained for each one of the numerical simulations can be observed in Figure 4. 

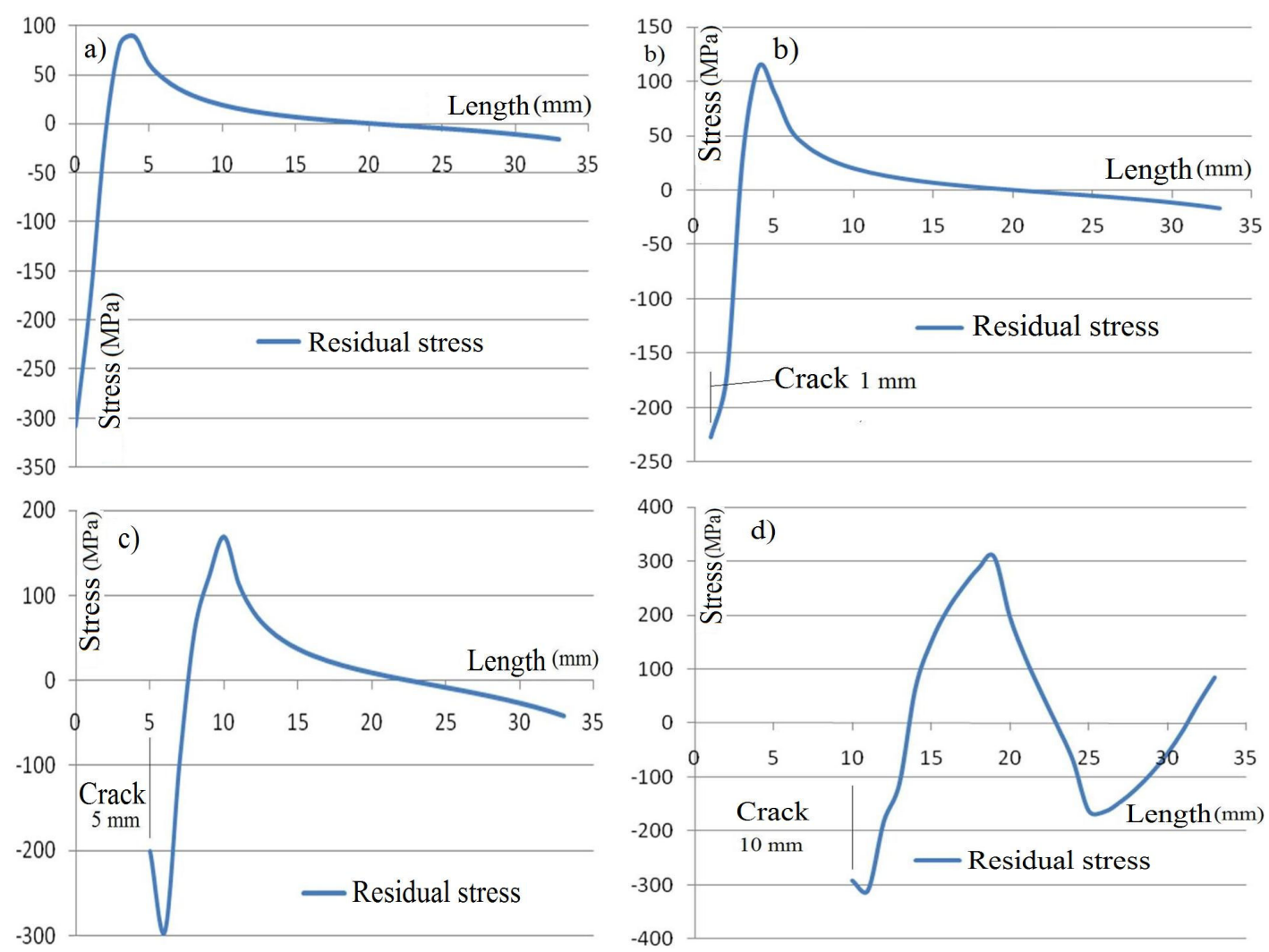

Figure 4.- Numerical evaluation of residual stress

a) Specimen without a crack. b) Specimen with a $1 \mathrm{~mm}$ crack length

c) Specimen with a $5 \mathrm{~mm}$ crack length. d) Specimen with a $10 \mathrm{~mm}$ crack length.
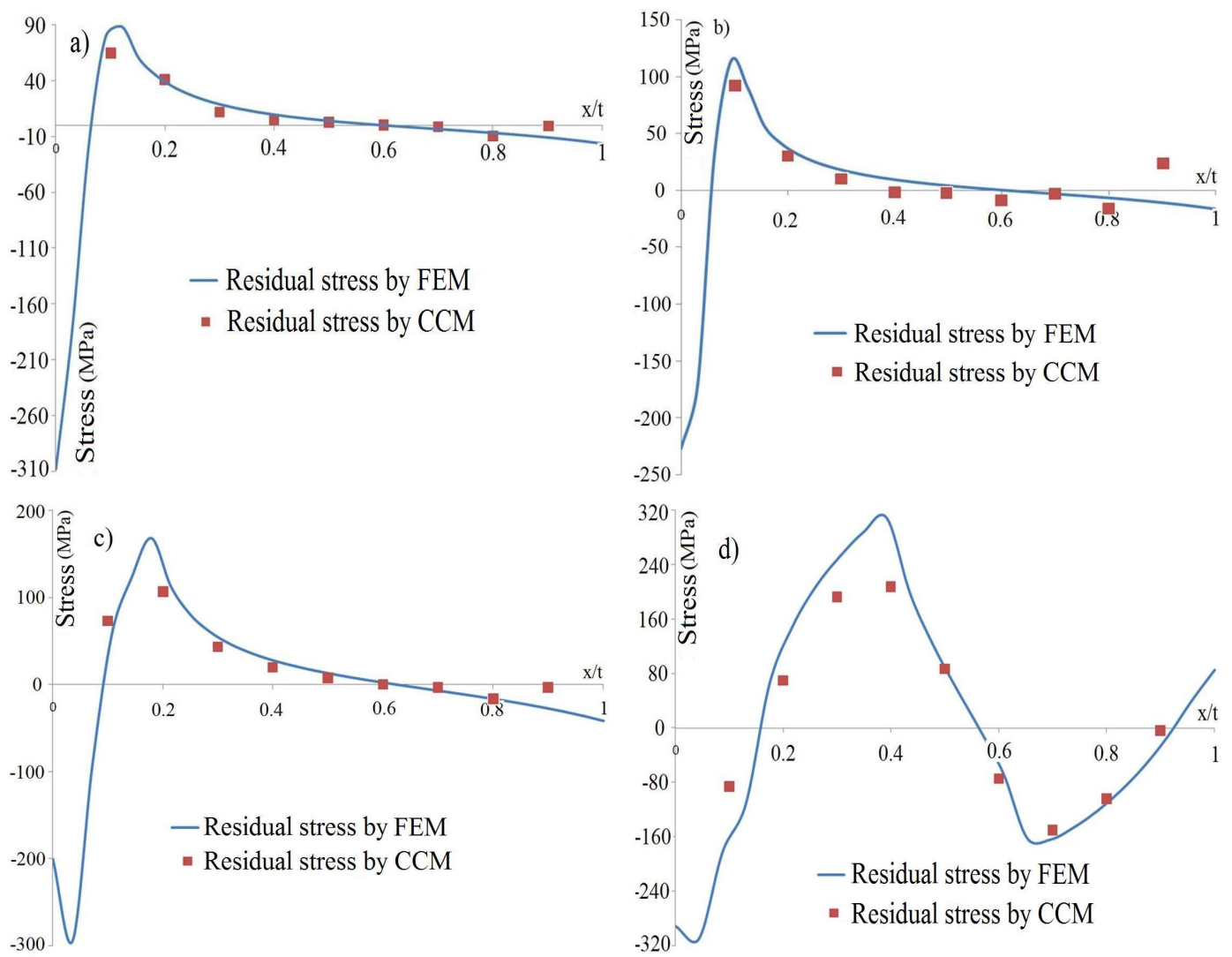

Figure 5.- Residual stress comparison between FEM and CCM

a) Specimen without a crack. b) Specimen with a $1 \mathrm{~mm}$ crack long c) Specimen with a $5 \mathrm{~mm}$ crack long. d) Specimen with a $10 \mathrm{~mm}$ crack long. 


\section{CCM numerical evaluation}

After the numerical simulation for the induction of the residual stress field was finished, a simulation of the behaviour of the $C C M$ was carried out. The theory and the way to apply the $C C M$ has been explained by several authors [7-9], also Urriolagoitia-Sosa, et al. have developed a new way to apply it [10]. The numerical evaluation of the $C C M$ was done by simulating the introduction of a slot, which will cause a modification by auto-equilibrium of the residual stress on the acting base line, producing a relaxation in the material. The relaxation will produce strain data, which can be used by the CCM to determine the acting residual stress field. The residual stress results obtained by the $C C M$ for each one of these cases is presented in Figure 5. In these figures there are shown as well comparisons against the numerical simulation by FEM.

\section{Conclusions}

This research was performed to validate the use of FEM in the introduction of residual stress and to validate its application to the $C C M$. The numerical data obtained will facilitate the experimental procedure for the induction of residual stress fields and the application to the $C C M$.

The modification of the compact tensile specimen $S E N$, will provide a more controllable set up for the introduction of residual stresses, this is because, if the specimen is kept with a notch tip, it cannot ensure the nucleation of a crack. The main idea of this research was to evaluate the effect of a crack in the introduction of residual stress fields, which has to be done by controlling (in certain degree) that no defects are present.

From Figure 4 it can be concluded, that after the effect of the load is removed from the specimens with a crack, a beneficial residual stress field has been induced. So, to propagate the crack it is required sufficient energy to overcome the residual stress field introduced into the component. Apparently, the combination of loading the crack and removing the load effect allows the material to raise its mechanical resistance. Nevertheless, much care has to be taken into consideration, because a crack is present in the specimen and could accumulate plastic energy due to subsequent loading cycles and then propagate.

Additionally, the exactitude on the application of the $C C M$ has been evaluated by the use of FEM. Significant data has been obtained for the experimental procedure of the CCM and the proper manner to establish its application. On the other hand it can be observed in Figure 5, that similar residuals stress fields between FEM and $C C M$ have been achieved. Nonetheless, it can be observed in Figure 5, that both ends of the curve for the calculated residual stress field are not as accurate as in the middle part of the specimen. It is thought that the mismatch observed at the opposite end of the rear location is due to the remote position in which the relaxation effect is evaluated, as the strain relaxation location cannot be fully determined. With respect to the discrepancy in residual stress results at the end near to the strain relaxation location it has been concluded, that this originates by the fact that the structural integrity of the material has been compromised by the introduction of the cut, and there is only a small part of material left.

\section{Acknowledgement}

The authors gratefully acknowledge the financial support from the Mexican government by the Consejo Nacional de Ciencia y Tecnología and the Instituto Politécnico Nacional. 


\section{References}

[1] D. Broek: Elementary engineering fracture mechanics (Martinus Nijhoff Publishers, Netherlands, The Hague 1984), p. 24-31.

[2] M.E. Fine and D.L. Davidson: in Fatigue Mechanisms, Advances in Quantitative Measurement of Physical Damage, ASTM STP 811, J. Lankford, D.L. Davidson, W.L. Morris, and R.P. Wei, eds., ASTM, Philadelphia, PA, 1983, p 350-70.

[3] G. Urriolagoitia-Sosa, J.F. Durodola and N.A. Fellows: Effect of strain hardening on residual stress distribution in beams determined using the crack compliance method, J. Strain Analysis for Eng. Design, Vol. 42, No. 2, 2007, p. 115-121.

[4] G. Urriolagoitia-Sosa: Analysis of prior strain history effect on mechanical properties and residual stress in beams, $\mathrm{PhD}$ thesis, Oxford Brookes University.

[5] E.A. Badr: A modified compact tension specimen for the study of residual stress maintainability, Experimental Techniques, May/June, 2000, p. 25-27.

[6] G. Urriolagoitia-Sosa, J.F. Durodola and N.A. Fellows: A method for the simultaneous derivation of tensile and compressive behavior of materials under Bauschinger effect using bend tests, Proceeding of the I Mech E, Part C, Journal of Mechanical Engineering Science, Vol. 220, No. 10, 2006, p. 1509-1518.

[7] W. Chang and I. Finnie: An overview of the crack compliance method for residual stress measurement. In Proceeding of the Fourth International Conference on Residual Stress (Society for Experimental Mechanics, Bethel, Connecticut) 1994, p. 449-458.

[8] M.B. Prime: Residual stress measurement by successive extension of a slot; The Crack Compliance Method, Applied Mechanics Reviews, Vol.52, No. 2, 1999, p. 75-96.

[9] H.J. Schindler: Residual stress measurement in cracked components: Capabilities and limitations of the cut compliance method, Materials Science Forum; Residual Stress ECRS 5, Vol. 347-349, 2000, p. 150-155.

[10] G. Urriolagoitia-Sosa, J.F. Durodola and N.A. Fellows: Determination of residual stress in beams under Bauschinger effect using surface strain measurements, Strain, Vol. 39, No. 4, 2003, p. 177-185. 